\title{
Network Scale Control for Distributed Networks by BER Analysis
}

\author{
Junyan Chen, Jing Liu, Yunfeng Guan, Wenlin Wang \\ Department of Electric Engineering \\ Shanghai JiaoTong University, Shanghai, 200240, China \\ Email:ericjunyan@yahoo.com.cn, \{jingliu_lj, yfguan69, wlwang009,\}@sjtu.edu.cn
}

\begin{abstract}
Node-to-node interference in the distributed networks comes to be one of the major challenges in recent wireless communication studies. The general methods make great improvement on such problem but influence the performances in other layer. It is because the models they based on, like SINR, barely consider the parameters across the layers. This paper adopts the network scale control to solve those problems. It not only can be developed to improve the interference challenge, such as subnet devision, but also can be combined with other algorithm or make references to engineering. In this paper, we discuss the network scale control according to a new interference model which yield the BER(Bit Error Rate) as result.The model considers the channel and topology of the network, and combine with the traffic and MAC policy across the layers. We take a typical distributed network for example and get the BER performance. We discuss the result of network scale control and compare the result with other methods.
\end{abstract}

Index Terms-Carrier-sensing Range, Network Scale, Cumulative Interference Model, BER, Cross Layer Design

\section{INTRODUCTION}

As far as the R\&D on the distributed networks (e.g. WLAN and WSN), people found more traffics and nodes are needed [1], especially in Ubiquitous Networks and Internet Of Things. Accordingly, it make node-to-node interferences increase significantly, and then seriously undermine the network performance. So that, the interference problem becomes one of the major challenges in the wireless fields.

So far, many methods have been proposed. MAC methods change the MAC policy [2], they avoid interferences with costs. Physic layer methods try to cop the interference by variable thresholds in modulation [3], it make sense in specific situation. Application methods adjust traffic [4] according to the interference, it is a compromise with the throughput.Bandwidth trade-off algorithms [5] and channel allocation methods [6] are also capable to improve the performance, but they have constrained source problems. Other methods like determining the communication region [7] are popularly used in engineering, however the range is substantially changeable when the parameters are changed. all of them make improvements in one layer, but they are exclude each other to build a synthetical system. It is found that the best solution is to consider the method across the layers [8].

This work was supported by International Collaborative Project of China Ministry of Science2010DFB10570, S2010GR0445 National Key Project of China (2010ZX0 3002-007-01)
Network scale is a nice point since it is a big factor to the interference [9], and hard to say which layer it belongs to because it relates to every layers. It is the basic condition of any models and it is more friendly and easy to combine with other algorithms. Controlling the network scale is one of the most useful methods in engineering and helpful to achieve other algorithms. Itself can also be developed to some kind of algorithm, such as subnet devision or multiple access.

To study on network scale needs to consider the interference model across layers. There are several interference models have been proposed already. The additive interference model [10], as known as SINR(Signal to Interference plus Noise Ratio) is the most famous one. It propose the SINR to take place of SNR to describe the network performance. But the SINR itself is little vague to measure the network performance and it is hard to transform into BER or network capacity. Other model like protocol model in [11], only is accurate when the transmitter is close enough to the receiver. The interference region model is found in [12], it is simplistic with a constant minimum interference range for all values.

The interference model in this paper uses BER as result to figure out the performance in the network. It treats each part in different layers as a component in the framework. Each component can be changeable according to the policy and protocol. The mainly contribution is presented in several parts:

- The network scale control method is introduced to make improvements in the network with interferences.

- A new interference model is designed across the layers.

- The network scale control is compared with other parameter control methods by BER performance.

The rest of the paper is organized as follow. We begin in Section II with a description of the new statistical interference model. We discuss the results and compare the network scale control with other parameter control methods in Section III and conclude in Section IV.

\section{Interference Model For Network SCALE CONTROL}

Every components in this network can be changed according to the cases. In order to introduce the interference model, we choose a typical network for example. It is a two-dimension distributed network of which the nodes are fixed in a square area. There is only one channel in this network and we assume that it is in a common office. We choose slotted Aloha in MAC 
and BPSK for modulation. For simplification, we assume the packets were aligned at transmitter. The power and the traffic of the nodes are equal for fairness. We introduce each component of the model as follows.

\section{A. The Topology}

We discuss a two-dimension network in a square area. Other shape like rectangle or hexagon are also available [13]. Generally, assume the distribution of nodes is uniform. According to that, we get the distribution of the distance between any nodes. We pick two nodes in this area, they might be $\mathrm{A}\left(x_{1}, y_{1}\right)$ and $\mathrm{B}\left(x_{2}, y_{2}\right)$. $\mathrm{A}$ and $\mathrm{B}$ are totally independent. So take A for example, p.d.f of the position should be:

$$
\begin{cases}f\left(x_{1}\right)=1, & 0<x_{1}<1 \\ f\left(y_{1}\right)=1, & 0<y_{1}<1\end{cases}
$$

So does node $\mathrm{B}$. Then, put $\mathrm{X}=\left|x_{1}-x_{2}\right|$ and $\mathrm{Y}=\left|y_{1}-y_{2}\right|$. The p.d.f is given by:

$$
\begin{cases}f(X)=2(1-X), & 0<X<1 \\ f(Y)=2(1-Y), & 0<Y<1\end{cases}
$$

$\mathrm{X}$ and $\mathrm{Y}$ are independent, so the joint p.d.f is:

$$
f(X, Y)=4(1-X)(1-Y), \quad 0<X<1,0<Y<1
$$

Since the distance between $\mathrm{A}$ and $\mathrm{B}$ is $r=\sqrt{X^{2}+Y^{2}}$, p.d.f of $r$ is:

$$
f(r)=d_{r}\left(\iint_{\sqrt{X^{2}+Y^{2}} \leq r} 4(1-X)(1-Y) d X d Y\right)
$$

Shift Cartesian to polar coordinates.Put $\theta=\arctan (Y / X)$ , then the p.d.f changes to:

$$
f(r, \theta)=4 r(1-r \cos \theta)(1-r \sin \theta)
$$

Integrating $f(r, \theta)$ respect to $\theta$ over appropriate limits and discussing $r$, the p.d.f of distance $r$ is found as:

$$
f(r)=\left\{\begin{array}{lc}
2 \pi r+2 r^{2}(r-4) & 0<r<1 \\
4 r\left(\arcsin r^{-1}-\arccos r^{-1}\right) & \\
+8 r \sqrt{r^{2}-1}-2 r\left(r^{2}+2\right) & 1<r<\sqrt{2} \\
0 & \text { else }
\end{array}\right.
$$

\section{B. Wireless Channel Model}

Since the nodes are fixed and there is no shadow. We use the following model to describe the large scale decline and multipath effect.

$$
P_{R}(r)=P_{T} G_{T} G_{R}\left(\frac{\lambda X}{4 \pi r}\right)^{n}=\frac{C}{r^{n}}
$$

Where $G_{r}$ and $G_{t}$ denote antenna gain which will be unity. $\lambda$ represents the wave length. $\mathrm{r}$ means the distance between two nodes. $\mathrm{n}$ represents to the pathloss exponent. $\mathrm{X}$ is an additional variable to describe the environment.
We assume that the network is in an office. The channel model is widely used to describe an indoor channel environment [14]. It is regarded as the developped model of the free space pathloss model [15]. In free space $n=2, X=1$. When the network is in office, the parameters should be measured. we measure a common office and get that $n=2.37, X=$ $e^{-10}$. Substitute (7) into (6), the single interference p.d.f is shown as follows:

$$
f_{I}(x)= \begin{cases}\frac{1}{n C}\left(\frac{x}{C}\right)^{-\frac{n+1}{n}}\left[2 \pi\left(\frac{x}{C}\right)^{-\frac{1}{n}}\right. & \\ \left.+2\left(\frac{x}{C}\right)^{-\frac{2}{n}}\left(\left(\frac{x}{C}\right)^{-\frac{1}{n}}-4\right)\right] & C<x<\infty \\ \frac{1}{n C}\left(\frac{x}{C}\right)^{-\frac{n+1}{n}}\left[4\left(\frac{x}{C}\right)^{-\frac{1}{n}}\right. & \\ \left(\arcsin \left(\frac{x}{C}\right)^{\frac{1}{n}}-\arccos \left(\frac{x}{C}\right)^{\frac{1}{n}}\right) & \\ +8\left(\frac{x}{C}\right)^{-\frac{1}{n}} \sqrt{\left(\frac{x}{C}\right)^{-\frac{2}{n}}-1} & 2^{-\frac{n}{2}} C<x<C \\ \left.-2\left(\frac{x}{C}\right)^{-\frac{1}{n}}\left(\left(\frac{x}{C}\right)^{-\frac{2}{n}}+2\right)\right] & \text { else } \\ 0 & \end{cases}
$$

\section{The Channel Access Model}

The adjacent nodes will provide interferences when they access to the channel. However, each nodes access to the channel with probability which connects to the traffic rate, the MAC police and the routing. We put the $P_{a}$ represents the probability of one node accessing to the channel. Since each node in the network is treated equally, we assume the $P_{a}$ for every nodes are same.

We suppose that each transmitter would have a buffer(or a queue).Thus, the transmitter can be regard as a M/M/1 system., the probability is:

$$
P_{a}= \begin{cases}\frac{\xi \lambda}{\mu} \cdot \frac{1}{N_{C}}=\frac{\xi \lambda}{\frac{B}{N_{C}}} \cdot \frac{1}{N_{C}}=\frac{\xi \lambda}{B}, & \frac{\xi \lambda}{\mu}<1 \\ \frac{1}{N_{C}}, & \frac{\xi \lambda}{\mu} \geq 1\end{cases}
$$

Where $\lambda$ denotes the traffic; $\xi$ represents the relay factor, it describes the additional relay traffic probably when the network allows multi-hops, then $\xi$ will be larger than 1 , so the arrival efficiency is $\xi \lambda$; the service efficiency is $\mu ; B$ means the data rate. $N_{C}$ denotes the average number of neighbors who are competing the channel. The $N_{C}$ is related to the relative sensing range and the number of nodes.

The attempt to the channel of each node is mutually independent. Assumed there are $\mathrm{M}$ nodes in the network,the probability of $m$ interference in the network and $b$ nodes are transmitting bit- 0 is:

$$
P\{m=k, b=l\}=\left(\begin{array}{c}
M \\
k
\end{array}\right)\left(P_{0} P_{a}\right)^{l}\left(P_{1} P_{a}\right)^{k-l}\left(1-P_{a}\right)^{M-k}
$$




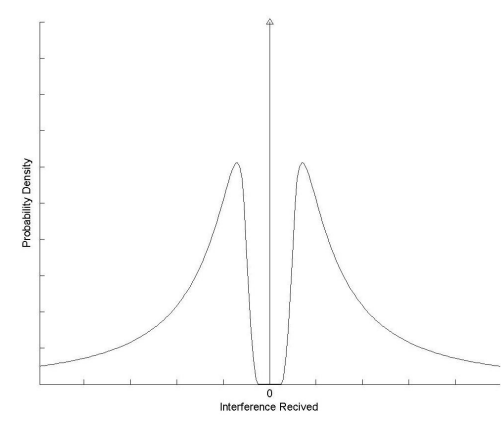

Fig. 1. The Profile of the Total Interference p.d.f

Where, the $P_{0}$ denotes the probability of sending 0 , likewise, $P_{1}$ denotes the one sends 1 . Statistically, $P_{0}=P_{1}=0.5$.

\section{The Total Interference in the Network}

The total interferences from $k$ nodes can be described as the combination of bit- 1 interferences and bit- 0 interferences:

$$
\hat{I}(t)=\sum_{i=0}^{l} I_{i}^{0}(t)+\sum_{j=0}^{k-l} I_{j}^{1}(t)
$$

Put $f_{\hat{I}}(x)$ as the p.d.f of the total interference one receiver got. We consider its characteristic function:

$$
\begin{aligned}
\varphi_{\hat{I}}(u)= & E\left[e^{i u \hat{I}(t)}\right] \\
= & E\left[e^{i u\left[\sum_{i=0}^{b} I_{i}^{0}(t)+\sum_{j=0}^{m-b} I_{j}^{1}(t)\right]} \mid m, b\right] \\
= & \sum_{k=0}^{M} \sum_{l=0}^{k} E\left[e^{i u\left[\sum_{u=0}^{b} I_{u}^{0}(t)+\sum_{v=0}^{m-b} I_{v}^{1}(t)\right]} \mid m=k, b=l\right] \\
& \cdot P\{m=k, b=l\}
\end{aligned}
$$

Since the interferences are mutually independent and with the same distribution, yield:

$$
\begin{aligned}
\varphi_{\hat{I}}(u) & =\sum_{k=0}^{M} \sum_{l=0}^{k}\left\{\prod_{u=1}^{l} E\left[e^{i u I_{u}^{0}(t)}\right] \prod_{v=1}^{k-l} E\left[e^{i u I_{v}^{1}(t)}\right]\right\} \\
& \cdot P\{m=k, b=l\} \\
& =\sum_{k=0}^{M} \sum_{l=0}^{k}\left[\varphi_{0}(u)\right]^{l}\left[\varphi_{1}(u)\right]^{k-l} P\{m=k, b=l\}
\end{aligned}
$$

Substitute (10) into (13), it is easy to get follows:

$$
\begin{aligned}
\varphi_{\hat{I}}(u)= & \sum_{k=0}^{M} \sum_{l=0}^{k}\left(\begin{array}{c}
M \\
k
\end{array}\right)\left(\begin{array}{c}
k \\
l
\end{array}\right)\left[\frac{1}{2} P_{a} \varphi_{0}(u)\right]^{l} \\
& \cdot\left[\frac{1}{2} P_{a} \varphi_{1}(u)\right]^{k-l}\left(1-P_{a}\right)^{M-k} \\
= & {\left[1-P_{a}+\frac{1}{2} P_{a} \varphi_{0}(u)+\frac{1}{2} P_{a} \varphi_{1}(u)\right]^{M} }
\end{aligned}
$$

Reverse (14) to the $f_{\hat{I}}(x)$, we get:

$$
\begin{aligned}
f_{\hat{I}}(x) & =\int_{-\infty}^{+\infty} \frac{e^{-i u x}}{2 \pi}\left[1-P_{a}+\frac{1}{2} P_{a} \varphi_{0}(u)+\frac{1}{2} P_{a} \varphi_{1}(u)\right]^{M} d u \\
& =\left[\left(1-P_{a}\right) \delta(x)+\frac{1}{2} P_{a} f_{I}^{0}(x)+\frac{1}{2} P_{a} f_{I}^{1}(x)\right]^{(M)}
\end{aligned}
$$

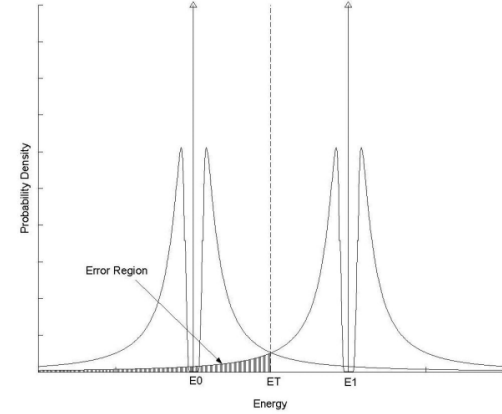

Fig. 2. The Profile of the Decision in BPSK

Where, $\delta(x)$ represents the Dirac function. It should be stressed that the superscript $(M)$ means $M$ th convolution.

\section{E. Modulation and Decision Model}

The BER performance relies on the modulation. We take BPSK for example. We have assumed that the packets are aligned at transmitters. Because the propagation distance is significant larger than the wave length and only half-wave loss happens in the propagation. We yield that the every half-bit are aligned at receivers.

Thus, signal and interferences overlapped, none or half phase difference. Bit-1 interferences and bit-0 interferences provide positive or negative impact on the signal respectively. The magnitude of such facilitation or neutralization by interferences are random, it relies on the p.d.f in (8). Since the probability of bit- 0 or bit- 1 are even and the half-wave loss effect occurs independently, the probability that interference is positive or negative at receivers is equal. The total interference p.d.f based on BPSK is finally presented as follow:

$$
f_{\hat{I}}(x)=\left[\left(1-P_{a}\right) \delta(x)+\frac{1}{2} P_{a} f_{I}(-x)+\frac{1}{2} P_{a} f_{I}(x)\right]^{(M)}
$$

Substitute (8) into (16). The function profile is shown in Fig.1. According to the (16), the p.d.f is an even function, and the arrow at the zero place represents the Dirac function of which the magnitude is meaningless, but the integration denotes the probability of none sending interference in the network at that moment. According to the p.d.f (16), the threshold is determined as Fig.2. The BER is calculate by integration beginning with the $E_{T}$.

\section{Performance Analysis And COMParison}

According to the model and substitute the variables in Table I, we yield the BER performance of network scale control. In order to give the explicit analysis, we discuss the network scale control and compare it with other parameter control methods.

\section{A. BER Performance of Network Scale Control}

First, we discuss the BER result of network scale control. Besides the parameters mentioned above, we increase the network scale from 1 node to 1000 nodes. We discuss network in a common office. The channel influence is not only presented by the distance between the nodes, but the signal also will be absorbed or reflect or refract by ceiling, floors, walls and 
TABLE I

PARAMETERS AND VARIABLES

\begin{tabular}{c|c|c}
\hline \hline Layer & Parameters & Value \\
\hline APL & Traffic $\lambda(\mathrm{kbps})$ & 10 \\
\hline NWK & Max. Hops Number $N_{h}$ & 3 \\
\hline MAC & Policy & Slotted Aloha \\
\hline PHY & Data Rate $B(\mathrm{Mbps})$ & 10 \\
& Modulation & BPSK \\
& Power & unification \\
& Antenna Gain $G_{T} / G_{R}$ & $1 / 1$ \\
& Frequency $(\mathrm{GHz})$ & 2.4 \\
\hline Others & Room Size $(\mathrm{m} \times \mathrm{m})$ & $100 \times 100$ \\
& Pathloss Exponent $n$ & 2.37 \\
& Separator Factor $X$ & $e^{-10}$ \\
\hline \hline
\end{tabular}

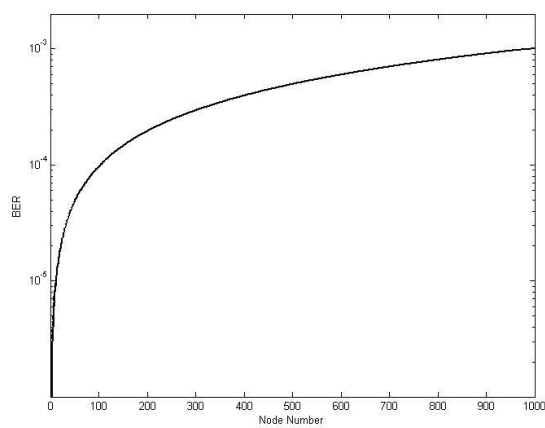

Fig. 3. The BER Performance of Two Different Wireless Channel, where in Common Office $\mathrm{n}=2.37, \mathrm{X}=e^{-10} ; \lambda=1 \mathrm{kbps}, R_{C}=0.1, R_{S}=0.1$

other separator.The network scale control result is shown in the Fig.3.

According to the Fig.3, the BER is small when the nodes in the network are less than about 100. It is because the expectation of the total interferences at that time is smaller than the signal. However the BER is raising very fast in this region, since every addition nodes make the total interferences more comparable with the signal. When the number of nodes reaches 300 , the BER becomes large since the total interferences is much larger than signal. And because of that, the addition of another node only makes a tiny different on interferences. The BER increases very slow at that time.

\section{B. Network Scale Control vs. Communication Range Control}

When the communication range shrinks, only closer nodes can be touched. The deserved signal power becomes bigger comparing to the interferences. The communication range control methods use this theorem. Now we compare it with network scale control.

To show the results explicitly, we shows the worst BER in the results. It means it is the worst case analysis and also can be thought as the receivers just locate at the rim of the communication range. Specially, it is used the relative size to describe the communication range here. It is relative to area size. The results are shown in Fig.4.

In Fig.4, it is shown that the BER is increasing when the network scale becomes larger, no matter what communication

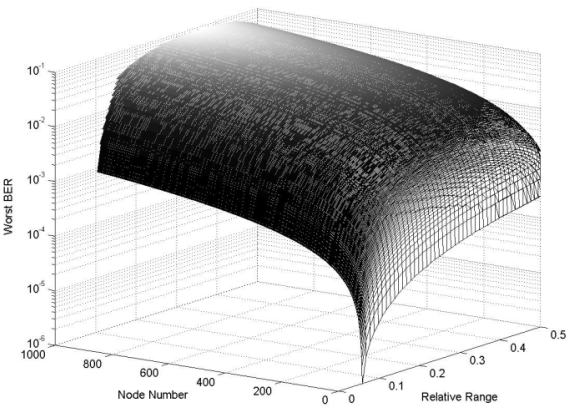

Fig. 4. The BER Performance vs. Network Scale \& Relative Communication Range, $\lambda=10 \mathrm{kbps}, R_{C}=R_{S}, N_{h}=3$

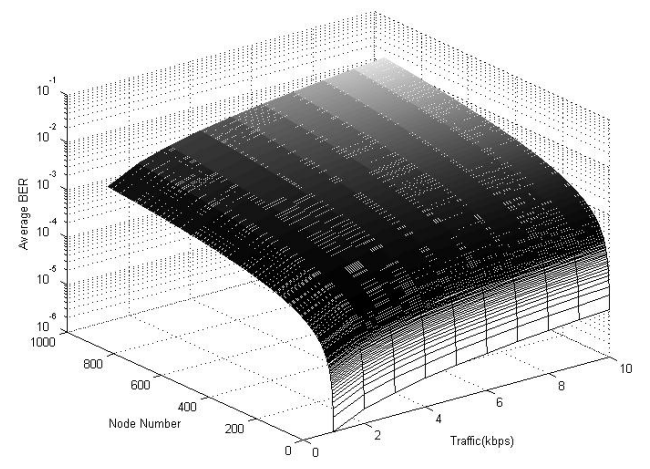

Fig. 5. The BER Performance vs. Network Scale \& Traffic, $R_{C}=$ $R_{S}=0.1, N_{h}=3$

range is. In the other hand, when communication range becomes larger, two effects make affect on the BER. As the range is extended, further receivers are reached. Since the distance causes the decline of the signals, the worst BER becomes large. At the same time, when communication range is spreading, the sensing range in MAC layer is extending as well, which causes the transmitter more sensitive to detect the collisions. More adjacent nodes have discovered the communication pairs and they keep silence. That reduces the interferences and decreases the BER. When the communication range is small, the adjacent nodes are litter, so the BER is dominated by transmitting distance. When range comes bigger, more nodes can detect the communication, the MAC policy dominates the BER performance. The MAC effect is invisible when the network scale is too small. It comes obvious when when the node number is large.

\section{Network Scale Control vs. Traffic Control}

The increasing traffic raises bigger probability of channel accesses so that the total interference will increase. We observe the BER performance when the traffic control versus network scale control in Fig.5.

In Fig.5, growth of the traffic raises the BER. According to the (9), when the traffic becomes heavy enough, the probability of the channel access comes to be a constant. It seems that the traffic make the BER change very slowly and almost no improvements when the traffic is heavy. It shows the traffic control is less efficiency than the network scale control does. 


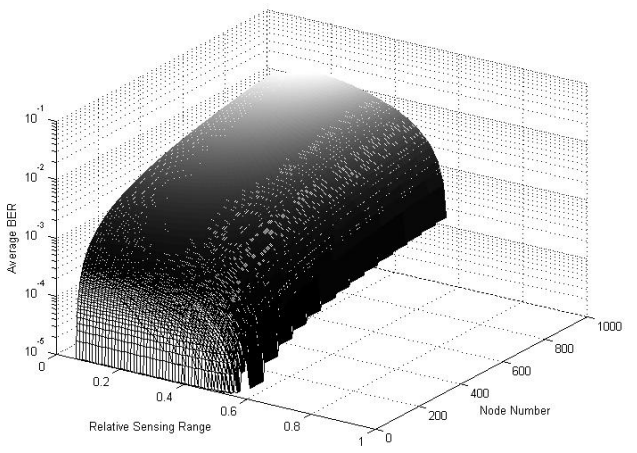

Fig. 6. The BER Performance vs. Network Scale \& Relative Sensing Range, $\lambda=10 \mathrm{kbps}, R_{C}=0.1$

\section{Network Scale Control vs. MAC Strategy}

There are so many kinds of MAC Strategies, we take one for example. The motive of the MAC strategy is to avoid the collisions. One way to do that is to reduce the collision threshold also known as extending the sensing range. It makes the transmitter able to detect more neighbors so that it can avoid more collisions. Fig. 6 shows the BER performance when the sensing range control versus network scale control.

It is obvious that the sensing control makes greater effect than network scale control. But the cost of that is throughput. Fig.7 shows the throughput in that situation. When sensing range reaches $60 \mathrm{~m}$, the BER decreases severely. It is because when the sensing range covers almost all the area, which means that the behaviors of every nodes is visible to each other, the BER in this case will comes to be zero. At same time, only one traffic is executed, which makes the throughput in this network extremely small. In the other hand, the network scale control may not reduce the BER that much, but the throughput in this way is stable.

\section{CONCLUSION}

We have studied on the network scale control to improve performance in network with interference. First we introduce a statistical framework for interference model in which each layer has been concerned. We get the topological model by discussing the a two-dimension fixed-node distributed network. Then we use a popular channel model to describe the single interference. Then we discuss the probability of the channel access by considering the MAC layer policy, routing factor and traffics. We consider other parameters across the layers to get the new interference model.

We use the model to analyze the BER performance of network scale control. We discuss the method in a typical office, and find the network scale control can improve the BER performance very well. We also compare the network scale control with other methods. Scale Control performs better than the traffic control,and almost even with communication range control, but the range control may raise sensing problem. The sensing range control shows it will improve the BER performance significantly, as prise, it will also reduce the throughput which will not happen to the network scale control.

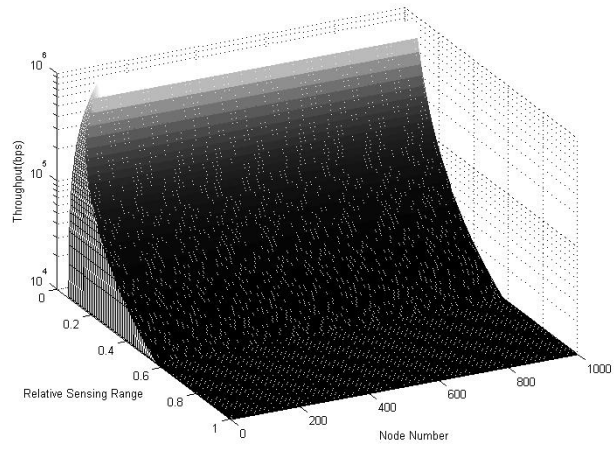

Fig. 7. The Throughput vs. Network Scale \& Relative Sensing Range, $\lambda=10$ kbps, $R_{C}=0.1$

We found the network scale control is useful and helpful to other methods. But it still has limitation, especially the MAC policy and routing. The routing protocol is only described as a factor, there is wider developing space from this angle, And more complicate MAC policy can be discussed in the further work. We may also develop the model by making the nodes movable or considering the details about the multipath.

\section{REFERENCES}

[1] Stavros Toumpis and Andrea J. Goldsmith, "Capacity Regions for Wireless Ad Hoc Networks," IEEE Transactions on Wireless Communications, Vol. 2, No. 4, July 2003.

[2] Stepan Kucera, Bing Zhang, "Predictive Techniques for Enabling Fast and Accurate Medium Access Control in Distributed Power-Controlled Networks," IEEE Transaction on Signal Processing, Vol. 57,No.8. 2009, pp2903-2907.

[3] Sang-Do Lee and Young-Chai Ko, "Joint Variable Threshold M-QAM and ARQ for Nakagami-m Fading Channel with Feedback Delay," 2010 International Conference on Electronics and Information Engineering (ICEIE 2010), Vol.2, 2010, pp5-11.

[4] Min Xie and Martin Haenggi, "A Study of the Correlations Between Channel and Traffic Statistics in Multihop Network," IEEE Transaction on Vehicular Technology, Vol. 56,No.6.November 2007, pp3550-3562.

[5] Nihar Jindal, Jeffrey G.Andrews, etc, "Bandwidth-SINR Tradeoffs in Spatial Networks,'ISIT2007, Nice, France, June 24 - June 29, 2007,pp836840 .

[6] Stepan Kucera, Sonia Aissa etc, "Adaptive Channel Allocation for Enabling Target SINR Achievability in Power-Controlled Wireless Networks," IEEE Transaction on Mobile Computing, Vol.9,No.2.February 2010, pp833-843.

[7] Hajar Mahdavi-Doost, Masoud Ebrahimi,etc, "Characterization of SINR Region for Interfering Links With Constrained Power," IEEE Transaction on Information Theory, Vol.56,No.6.June 2010, pp2816-2818.

[8] Mehmet C. Vuran and Ian F. Akyildiz, "XLP: A Cross-Layer Protocol for Efficient Communication in Wireless Sensor Networks," IEEE Transactions on Mobile Computing, Vol.9, No.11, November 2010

[9] V.R. Cadambe and S.A. Jafar, "Interference alignment and degrees of freedom of the K-user interference channel," IEEE Transactions on Information Theory, Vol.54,No.8, August 2008, pp. 3425-3441.

[10] A. Karnik, A. Iyer, and C. Rosenberg,"Throughput-optimal configuration of wireless networks," IEEE/ACM Trans. Networking,Vol.15,No.5, 2008.pp1161-1174.

[11] P. Gupta and P. R. Kumar,"The capacity of wireless networks," IEEE Trans. Inform. Theory, Vol.IT-46, No.2, pp.388-404, Mar.2000.

[12] T. Nandagopal, T.E. Kim,etc,"Achieving MAC layer fairness in wireless packet networks," in Proc. ACM/IEEE MobiCom 2000, Aug. 2000.

[13] Pingyi Fan, Guansheng Li,etc, "On the Geometrical Characteristic of Wireless Ad-Hoc Networks and its Application in Network Performance Analysis," IEEE Trans.on Wirless Communications, vol.6, NO.4, 2007.

[14] Claude Oestges, Danielle V.Janvier,etc, "Channel Characterization of Indoor Wireless Personal Area Networks," IEEE Transaction on Antennas and Progagation, Vol.54, No.11, November 2006.

[15] T.S. Rappaport, Wireless Communications, Prentice Hall Press, 1996. 